\title{
Diseño de software para calcular la huella de carbono e hídrica durante la producción de café
}

\author{
Design of software to calculate the carbon \\ and water footprint during coffee production
}

\author{
Irlesa Indira Sánchez-Medina ${ }^{1} \bowtie$, Ferley Medina-Rojas ${ }^{2}$, \\ Jaime Malqui Cabrera-Medina ${ }^{3}$
}

Universidad Cooperativa de Colombia, sede Neiva, Huila, Colombia
${ }^{2}$ Universidad Cooperativa de Colombia, sede Neiva, Huila, Colombia
${ }^{3}$ Universidad Cooperativa de Colombia, sede Neiva, Huila, Colombia

Calle 11 N. ${ }^{0}$ G $\mathrm{G}$-31. Programa de Ingeniería de Sistemas, Universidad Cooperativa de Colombia, Neiva, Colombia.

Email: Irlesa.Sanchez@campusucc.edu.co

Recibido: 10 de septiembre del 2017 Aprobado: 1 de diciembre del 2017 Disponible en línea: 1 de enero del 2018

How to cite this article: I. I. Sánchez-Medina, F. Medina-Rojas, y J. M. Cabrera-Medina, "Diseño de software para calcular la huella de carbono e hídrica durante la producción de café”, Revista Ingeniería Solidaria, vol. 14, no. 24, pp. 12, enero 2018. doi: https://doi.org/10.16925/in.v14i24.2159

\section{Resumen}

Introducción: el artículo es producto de la investigación "Diseño de software para calcular la huella de carbono e hídrica durante la producción de café", desarrollada en la Universidad Cooperativa de Colombia, sede Neiva, en el periodo 2016-2017.

Objetivo: diseñar un software para el cálculo de la huella de carbono e hídrica en la producción de café. Metodología: el desarrollo del proceso de investigación es de carácter cualitativo y cuantitativo, con reconocimiento de conceptos, contextos y experiencias de los agricultores de café en los municipios de Acevedo, San Agustín, San José de Isnos y Pitalito.

Resultados: el diseño de una aplicación web para calcular huellas de carbono y huella hídrica durante el cultivo de café, la cual integre tecnologías bajo licencia de software libre que evidencien eficiencia y eficacia en el desempeño.

Conclusión: la metodología xp garantiza un desarrollo ágil durante el diseño de software, lo que permitirá calcular la huella de carbono e hídrica adaptable a cambios y requisitos en corto tiempo y con un bajo costo.

Originalidad: hasta el momento, no se evidencia en el estado del arte un diseño del cálculo de huella de carbono e hídrica para la producción de café en el sur del Huila.

Limitaciones: este proyecto utilizó una metodología que se puede aplicar a diferentes cultivos; sin embargo, los cálculos de las huellas son específicas a estas zonas dadas las variables del medio ambiente.

Palabras clave: huella ambiental, huella de carbono, huella hídrica, software para huellas ambientales. 


\title{
Software Design for Carbon and Water Footprint Calculation in Coffee Production
}

\author{
Abstract \\ 2016-2017. \\ Aim: To design software for calculating carbon and water footprints in coffee production. \\ Pitalito. \\ effectiveness. \\ tion in coffee production in southern Huila. \\ calculations are specific to these areas given the environmental variables.

\section{Desenho de software para calcular a pegada de carbono e hídrica durante a produção de café}

Introduction: The article derives from the research "Software design for carbon and water footprint calculation in coffee production" conducted at the Universidad Cooperativa de Colombia, Neiva, in the period

Methods: The research process is both qualitative and quantitative, acknowledging opinions, contexts and experiences of coffee farmers in the municipalities of Acevedo, San Agustín, San José de Isnos and

Results: The design of a web application to calculate carbon footprints and water footprints in coffee growing, integrating technologies under a free software license that show performance efficiency and

Conclusion: The xp methodology ensures flexible development in software design, which will allow carbon and water footprint calculation adaptable to changes and requirements in a short time and at a low cost.

Originality: So far there is no evidence in the literature of a design for carbon and water footprint calcula-

Limitations: This project used a methodology that may be applied to various crops; however, footprint

Keywords: environmental footprint, carbon footprint, water footprint, environmental footprint software.

\section{Resumo}

Introdução: este artigo é produto da pesquisa "Desenho de software para calcular a pegada de carbono e hídrica durante a produção de café", desenvolvida na Universidad Cooperativa de Colombia, sede Neiva, no período de 2016 e 2017.

Objetivo: desenhar um software para o cálculo da pegada de carbono e hídrica na produção de café.

Metodologia: 0 desenvolvimento do processo de pesquisa é de carácter qualitativo e quantitativo, com reconhecimento de conceitos, contextos e experiências dos agricultores de café nos municípios de Acevedo, San Agustín, San José de Isnos e Pitalito (Colômbia).

Resultados: 0 desenho de uma aplicação web para calcular pegadas de carbono e hídrica durante o cultivo de café, a qual integre tecnologias sob licença de software livre que evidenciem eficiência e eficácia no desempenho.

Conclusão: a metodologia xP garante um desenvolvimento ágil durante 0 desenho de software, 0 que permitirá calcular a pegada de carbono e hídrica adaptável a mudanças e requisitos em curto tempo e com baixo custo.

Originalidade: até o momento, não tem sido evidenciado no estado da arte um desenho do cálculo de pegada de carbono e hídrica para a produção de café no sul de Huila (Colômbia).

Limitações: este projeto utilizou uma metodologia que pode ser aplicada a diferentes cultivos, contudo os cálculos das pegadas são específicos a essas zonas, tendo em vista as variáveis do meio ambiente.

Palavras-chave: pegada ambiental, pegada de carbono, pegada hídrica, software para pegadas ambientais. 


\section{Introducción}

En el cultivo del café, la siembra de cafetos se da por partes: primero, se siembran semillas o bayas en invernaderos controlados; luego de un tiempo, se obtiene el almácigo y se traslada al destino final del arbusto (el tiempo promedio para que un cafeto produzca café de calidad y de manera sostenida es de tres años, y produce café hasta por 50 años) [1]. Dado lo anterior, es importante aclarar que la huella ambiental que genera la industria del café incluye los procesos de producción, procesamiento y distribución. La huella de carbono (HC), por ejemplo, es un concepto que se desarrolló a fin de describir los balances entre remociones y emisiones de gases de efecto invernadero (GEI) que un determinado bien o servicio genera durante su ciclo de vida. Actualmente, este concepto se considera altamente relevante en la conciencia pública sobre cambio climático, lo que da lugar a un interés creciente por su estimación y declaración asociada con el análisis de ciclo de vida (ACV) de un producto [2]. Para el caso de la huella hídrica $(\mathrm{HH})$ en la producción de cafés especiales en ocho cuencas hidrográficas de cuatro municipios del sur del Huila, permite conocer los volúmenes de agua dulce consumido para la producción de café pergamino seco en los diferentes componentes de la huella hídrica total (ннт).

Según el Instituto Superior del Medio Ambiente, la huella de carbono se define como el cálculo del total de las emisiones de gases de efecto invernadero emitidas por un individuo, una organización, un evento o un producto de forma directa e indirecta. Una organización puede estar interesada en calcular su huella de carbono o la de sus productos y servicios por diversas razones:

- Mejorar la imagen de marca y la reputación frente a la competencia.

- Dar respuesta a exigencias de clientes, consumidores o inversores.

- Reducir los costes asociados al cumplimiento de la legislación al adaptarse precozmente a nuevos requisitos.

- Evaluar los riesgos asociados al cambio climático e identificar potenciales ahorros y oportunidades de acceso a nuevos mercados.

- Dar respuesta al compromiso de la organización con la planificación de programas efectivos de reducción de emisiones GEI [3].
La entidad Conservación y Carbono s. A. s. [4] presenta la huella hídrica con el propósito de mejorar procesos y abordar con un mayor entendimiento la gestión del agua, así como las problemáticas de escasez y contaminación de fuentes. En el estudio se incorpora toda la cadena de producción y distribución de un bien o un servicio, se genera un diagrama de todo el proceso y se identifican con precisión los consumos asociados a cada etapa. Considerar y disminuir la huella hídrica de su empresa es una estrategia dirigida a reducir riesgos de reputación (uso inadecuado del recurso), físicos (escasez del recurso), legales (mayores controles ambientales) y financieros (mayores costos operativos y menores ganancias). Así, se obtiene como beneficios:

- Ecoetiquetado en los productos y servicios.

- Análisis de la sostenibilidad del consumo del recurso agua.

- Se mejora la competitividad de los productos y servicios.

- Se mejora la imagen institucional al transmitir de manera clara un compromiso con el medio ambiente.

- Se detectan costos evitables asociados al consumo de agua.

- Se abre la posibilidad de optimizar procesos y operaciones.

- Se fortalece el cumplimiento del Decreto 1299 de 2008: funciones del Departamento de Gestión Ambiental Empresarial [4].

\subsection{Huella ambiental o ecológica}

La huella ambiental o ecológica es lo que requieren los seres vivos (fauna y flora) para crecer y vivir; en el caso de un individuo, ciudad o país, sin darse cuenta satisface su necesidad de consumo y absorbe residuos. La huella ecológica depende del estilo de vida. Un ejemplo de lo anterior es el siguiente: las personas que habitan en la ciudad desperdician grandes cantidades de agua puesto que utilizan aparatos electrónicos, consumen alimentos que son importados de otros países o regiones lejanas, se transportan en moto, carro y avión, utilizan demasiados envases plásticos y generan muchos desperdicios y basuras; al utilizar estos recursos se reducen las reservas naturales (los bosques, las selvas, los ríos y gran parte de los mares del mundo) [5]. 


\subsection{Huella de carbono}

Para la medición del nivel de emisiones de $\mathrm{CO}_{2}$ de acuerdo con una determinada actividad - bien la desarrolle un individuo o bien un grupo-, y clasificarla de diversas maneras, es necesario aplicar normativas ambientales internacionales como la IsO 14064, la PAS 2050, o la GHG Protocol, entre otras. Esta calificación sirve luego para determinar qué medidas pueden o deben tomarse en el propósito de disminuir el nivel de emisión y así reducir el impacto de dicha actividad en el ambiente. La tendencia del método de la huella de carbono es ubicar toda industria en el estado "carbono neutro", esto es, mediante políticas de disminución de emisiones y de limpieza atmosférica, remover de la atmósfera tanto $\mathrm{CO}_{2}$ como el que se emitió en las actividades de dicha industria, y eliminar de esta manera el impacto atmosférico [6]. Como parte de los métodos para calcular la huella de carbono, cabe mencionar los siguientes:

- Greenhouse gas protocol corporate standard (GHG Protocol). Desarrollado por Word Resources Institute (Instituto de Recursos Mundiales) y World Business Council for Sustainable Development (Consejo Empresarial Mundial para el Desarrollo Sostenible), es uno de los protocolos más utilizados a escala internacional para cuantificar y gestionar las emisiones de GEI.

- UNE-ISO 14064-1. De acuerdo con el GHG Protocol, se desarrolla en el 2006 la norma Iso 14064, la cual se estructura en tres partes. Para esta guía se aplicaría la 14064-1, puesto que especifica los principios y requisitos a nivel de organización para la cuantificación y el informe de emisiones y remociones de GEI. Las otras partes de esta norma se dirigen, por un lado, a proyectos sobre GEI específicamente diseñados con el fin de reducir las emisiones de GEI o aumentar la remoción de GEI (ISO 14064-2), y, por otro, a la validación y la verificación de los GEI declarados (Iso 14064-3).

- UNE-ISO 14065: 2012. Requisitos para los organismos que realizan la validación y la verificación de gases de efecto invernadero, en relación con su uso en acreditación u otras formas de reconocimiento.

- UNE-ISO 14069: 2013. Cuantificación e informe de GEI para organizaciones. Constituye la guía para la aplicación de la Iso 14064-1.
- IPCC 2006 GHG Workbook. Una completa guía para calcular GEI proveniente de diferentes fuentes y sectores; incluye una detallada lista de factores de emisión. Esta guía se creó con el fin de servir de orientación en la cuantificación de las emisiones de GEI de los inventarios nacionales, pero puede ser de gran utilidad a la hora de calcular la huella de carbono de las organizaciones. Si no se dispone de factores de emisión específicos, el "IPCC 2006 GHG Workbook" proporciona factores de emisión genéricos que pueden servir para calcular la HC de una organización.

- Bilan Carbone (Francia). La Agence del'Environnement et de la Maîtrise de l'Energie (Agencia Francesa del Medio Ambiente y Gestión de la Energía) elaboró e implementó a partir del 2004 esta herramienta metodológica dedicada a la medición de emisiones de GEI. Se basa en los contenidos del GHG Protocol y la Iso 14064

- Indicadores GRI (Global Reporting Initiative). Iniciativa internacional en la que participan entidades de diversos ámbitos, lo que incluye empresas, gobiernos y diferentes organizaciones civiles. Su objetivo es establecer un marco de trabajo común a nivel mundial con un lenguaje uniforme y parámetros comunes que sirvan para comunicar de una forma clara $y$ transparente las cuestiones relacionadas con la sostenibilidad a través de las denominadas "Memorias de sostenibilidad". Las mencionadas memorias comprenden información de diversa índole, entre la que se encuentran los indicadores de desempeño. Estos indicadores permiten disponer de información comparable con respecto al desempeño económico, ambiental y social de la organización.

- Recomendación de la Comisión de 9 de abril del 2013. Se dan sobre el uso de métodos comunes para medir y comunicar el comportamiento ambiental de los productos y las organizaciones a lo largo de su ciclo de vida (2013/179/UE).

- ISAE 3410. Norma internacional aprobada por el Consejo de Normas Internacionales de Auditoría y Aseguramiento (IAASB) en marzo del 2012; trata sobre contratos de aseguramiento de informes de gases de efecto invernadero.

En el cálculo de la huella de carbono, se toma como referencia: 1 . El factor de emisión $\left(\mathrm{kg} \mathrm{CO}_{2}\right.$ eq masa/volumen $/ \mathrm{kwh} / \mathrm{km}) ; 2$. Los datos de actividad (masa/volumen $\mathrm{kwh} / \mathrm{km}$ ); y 3. Huella de carbono 
$\left(\mathrm{kg} \mathrm{CO}_{2}\right.$ eq/unidad funcional $=$ datos de $\operatorname{actividad}^{*}-$ factor de emisión).

Cabe resaltar que las entidades que calculan huella de carbono contribuyen en la lucha contra el cambio climático, con la ventaja de identificar oportunidades de reducción de emisiones de GEI a fin de obtener ahorros económicos. Los esquemas voluntarios nacionales (registro de huella de carbono, compensación y proyectos de absorción de dióxido de carbono), regionales o privados identifican oportunidades de negocio: atraer inversionistas y clientes sensibilizados con el cambio climático y el medio ambiente [7].

La fase de análisis y diseño será pieza clave en el desarrollo del software que tiene como objetivo medir la huella de carbono y la hídrica durante la producción de café. Una vez alcanzado el objetivo, se propone contribuir a la reducción de desperdicios de recursos naturales tales como el agua, así como optimizar los procesos para la disminución de emisión de gases efecto de invernadero.

El departamento del Huila es líder nacional en el desarrollo de la caficultura y consolida su lugar como primer productor de café del país. De acuerdo con la Gerencia Técnica de la Federación Nacional de Cafeteros de Colombia - la cual se basa en el análisis del último año cafetero (octubre del 2014-septiembre de 2015) - , de una producción total de 13333000 sacos de café de 60 kilos, el departamento del Huila aportó el 17,99 \%, seguido de Antioquia con el 16,15\%, Tolima con el $12,74 \%$, Caldas con el 9,05 \% y Cauca con el 8,77 \% [8]. Para el 2016 (como se evidencia en la figura 1), la compra de café por parte de las cooperativas tuvo un repunte importante con grano estándar de 6205916 kilogramos, y en café especial a 10335986 kilogramos, para un total de 16541902 kilogramos, esto es, 5008385 kilos más con relación al mismo periodo del 2015 (la variación es entonces del 30,28 \%). Para el caso de los compradores particulares, el incremento fue un poco menor: durante el primer semestre del 2015 se adquieren 58524413 kilos de café, y este año, las compras subieron a 62674150 kilogramos (6,62 \% más de una vigencia a la otra). Se presenta un total entre las compras de las cooperativas y las de los particulares en el periodo de análisis el año pasado por el orden de los 70057930 kilos, y en el 2016 crecieron a 79216052 kilos, una variación del 11,56\% [9].
Tabla 1. Compra de café en el Huila

\begin{tabular}{|c|c|c|c|}
\hline \multicolumn{4}{|c|}{ Enero-junio (kilogramos) } \\
\hline Descripción & 2015 & 2016 & $\begin{array}{l}\text { Validación } \\
\text { absoluta }\end{array}$ \\
\hline Café estándar & 450395 & 6205916 & \\
\hline Café especial & 11083122 & 10335986 & \\
\hline $\begin{array}{l}\text { Total, recibido } \\
\text { por alamacafé }\end{array}$ & 11533517 & 16541902 & 5008385 \\
\hline $\begin{array}{l}\text { Café- } \\
\text { particulares }\end{array}$ & 58524413 & 62674150 & 4149737 \\
\hline Total, semestre & 70057930 & 79216052 & 9158122 \\
\hline
\end{tabular}

Fuente: [9]

\subsection{Huella hídrica}

La huella hídrica es un indicador de uso de agua dulce que es palpable no solo en el uso directo de agua de un consumidor o productor, sino también en su uso indirecto. Es un concepto muy reciente introducido en el 2002 por Arjen Hoekstra, experto del Instituto Unesco-IHe y profesor de la Universidad de Twente en los Países Bajos. La huella hídrica representa la cantidad de agua que hace falta para sostener la actividad de una población cuyos componentes pueden ser especificados geográfica y temporalmente [10]. Para el cálculo de la huella hídrica, se utiliza la metodología estándar propuesta por el Water Footprint Network, en la que define la huella hídrica de cualquier bien o servicio como el volumen de agua utilizado directa e indirectamente para su producción, sumados los consumos de todas las etapas de la cadena productiva. La huella hídrica de un individuo no está solo relacionada con su consumo directo de agua, sino con sus hábitos de vida. Se toman como referencia tres componentes básicos para el cálculo de la huella de agua [11]:

- Huella hídrica de un proceso. La huella hídrica como indicador multidimensional que sirve para diferenciar entre el agua consumida según el lugar de donde provenga y el agua contaminada, tal como se muestra en la fórmula de la huella hídrica de un proceso. Se distingue entre huella hídrica azul $\left(\mathrm{HH}_{\text {azul }}\right)$, verde $\left(\mathrm{HH}_{\text {verde }}\right)$ y gris $\left(\mathrm{HH}_{\text {gris }}\right)$; la suma de estos componentes constituye la huella hídrica de un proceso. 
La fórmula de la huella hídrica de un proceso es: $H H_{\text {proceso }}=H H_{\text {verde }}+H H_{\text {azul }}+H H_{\text {gris }}=H H_{\text {directa }}+$ $\mathrm{HH}_{\text {indirecta }}$.

- Huella del agua verde. La huella verde del agua $\left(\mathrm{HH}_{\text {verde }}\right)$ como indicador del uso es el volumen de agua de lluvia consumido durante el proceso de producción (véase la fórmula de la huella del agua verde). Se calcula a partir de la evapotranspiración total del agua de lluvia por los campos y las plantaciones, sumada al agua incorporada en la cosecha o en la madera cosechada. La fórmula de la huella del agua verde es: $H H_{\text {verde }}$ = evaporación del agua verde + incorporación del agua verde.

- Huella del agua azul. En el proceso de determinación de la $\mathrm{HH}_{\text {azul }}$ es posible distinguir entre diferentes fuentes de agua azul, tal como se presenta en la fórmula de la huella del agua azul. La división más utilizada es la que se realiza entre agua superficial, el agua subterránea renovable y la fósil. En la práctica, es a menudo muy difícil hacer la distinción debido a la falta de los datos. La fórmula de la huella del agua azul es: $H H_{\text {azul }}=$ evaporación del agua azul + incorporación del agua azul + flujo de vuelta perdido.

- Huella del agua gris. Para el caso de los productos químicos o un agua residual, estos se lanzan directamente en un cuerpo de agua superficial (la carga se puede medir directamente). La HGA se calcula al dividir la carga del agente contaminador ( $\mathrm{L}$ en masa/tiempo) por la diferencia entre el estándar ambiente de la calidad del agua para ese agente contaminador $\left(c_{\max }=\right.$ nivel aceptable máximo de concentración en masa/volumen), y su concentración natural en el cuerpo del agua de recepción ( $\mathrm{c}_{\text {nat }}$ en masa/volumen) o a partir del volumen de efluente (Ef), la concentración de agentes contaminantes en el efluente $\left(c_{\mathrm{ef}}\right)$, la concentración natural $\left(\mathrm{c}_{\text {nat }}\right)$ y la máxima permisible $\left(c_{\max }\right)$, tal como se presenta en la ecuación 1.

$$
H H_{g r i s}=\frac{V_{e f} x\left(C_{e f}-C_{n a t}\right)}{C_{e f}-C_{n a t}}
$$

En el caso de procesos agrícolas, con el uso de fertilizantes o de pesticidas, cuando el producto químico se aplica directamente en el suelo, puede suceder que solo una fracción ( $\alpha$ ) se filtre en el agua subterránea o se escurra sobre la superficie a una corriente del agua superficial. En este caso, la carga del agente contaminador es la fracción de la cantidad total de productos químicos aplicados (AR) que alcanza el agua de tierra o superficial. La concentración natural $\left(c_{\text {nat }}\right)$ en un cuerpo del agua de recepción es la concentración en el cuerpo del agua que ocurriría si no hubiera disturbio humano en la captación y el estándar ambiente de la calidad del agua para ese agente contaminador $\left(\mathrm{c}_{\max }=\right.$ nivel aceptable máximo de concentración en masa/volumen) [12] [13].

$$
\mathrm{HH}_{\text {gris }}=\frac{\alpha \times \mathrm{AR}}{C_{\max }-C_{n a t}}
$$

\subsection{Software para huellas ambientales}

A fin de calcular la huella de carbono, existen diversas herramientas que permiten conocer los impactos ambientales que generan los productos y organizaciones en el planeta. A continuación, se relacionan algunas herramientas en línea:

- La calculadora de huella ecológica es una herramienta en línea que sirve para medir y evaluar el estilo de vida que la persona tiene sobre el planeta en relación con la capacidad de la naturaleza para renovar sus recursos [14].

- La empresa Ecopetrol dispone de una herramienta denominada "Calcule su huella de $\mathrm{Co} 2$ ", con el objetivo de generar conciencia en la comunidad sobre cuánto se contamina y qué medidas se deben tomar al respecto [15].

- Para el caso de grandes empresas, se encuentra el software Air.e LCA y Air.e Hdc de Solidforest, un software de análisis de ciclo de vida (AVC) para el estudio del comportamiento ambiental de productos y organizaciones. Con él se podrá elaborar la huella ambiental promovida por la Unión Europea, desarrollar declaraciones ambientales de producto (DAP) y Ecodiseño, así como calcular huella de carbono, huella hídrica o huella de agua de productos, servicios y organizaciones [16].

\section{Metodología}

Se utilizó una metodología de investigación cualitativa y cuantitativa de tipo investigación acción 
participativa basada en el reconocimiento de la experiencia de los agricultores de café durante el proceso de producción. Involucra diferentes variables para utilizar en el diseño del software y desarrollar los cálculos necesarios con metodología XP. La anterior metodología será la variable principal durante el diseño de software para determinar huellas ambientales (carbono e hídrica) durante la producción de Café, y se desarrollará en dos fases.

\subsection{Fase 1: análisis y diseño}

Para el análisis, se toma como referencia una muestra de 500 caficultores a los cuales se aplicaron protocolos de observación e información documental; se realizaron encuentros con ellos con el fin de identificar variables involucradas durante la producción de café. En la fase del diseño, se toma como referencia la metodología XP (es el enfoque de desarrollo de sistemas que controla el tiempo, el costo, la calidad y el alcance, y cuyas actividades se centran en codificar, probar, escuchar, diseñar y alcanzar una entrega oportuna) [17]. Con esta metodología, también se podrá realizar el diseño del modelado de datos, el diseño de la base de datos y el diagrama de distribución necesarios para el desarrollo del software.

Las historias de usuario es la técnica utilizada en la metodología XP para especificar los requisitos del software. El cliente describe características que el sistema debe contener como requisito funcional o no funcional. Las historias de usuario son dinámicas y flexibles, de modo que en cualquier momento pueden romperse o reemplazarse por otras específicas o generales, así como añadir nuevas o modificarlas. Cada historia de usuario se concibe y delimita con el fin de que los programadores puedan implementarla en pocas semanas, tal como se presenta en la tabla 2 .

Además, la plataforma debe contar con los desarrollos que permitan realizar los cálculos de huellas ambientales y los algoritmos propuestos para determinar alertas tempranas de floración, producción, alertas de broca, roya, renovación, fertilización y épocas oportunas de siembra.

\subsubsection{Personal requerido/roles}

- Gerente de proyecto. Realizar su seguimiento y ejecución dentro de los plazos establecidos.
Tabla 2. Historias de usuario n. ${ }^{\circ} 1$

\begin{tabular}{|c|c|c|}
\hline N. ${ }^{\circ}$ & Historia de usuario & $\%$ \\
\hline 1 & $\begin{array}{l}\text { Datos de los cuatro municipios del sur del } \\
\text { departamento del Huila }\end{array}$ & 2 \\
\hline 2 & Establecer las variables a utilizar & 2 \\
\hline 3 & $\begin{array}{l}\text { Establecer personal requerido con sus respec- } \\
\text { tivos roles }\end{array}$ & 2 \\
\hline 4 & Establecer esquemas de restricción & 3 \\
\hline 5 & Establecer interfaces de entrada y salida & 3 \\
\hline 6 & Establecer el catálogo de elementos necesarios & 3 \\
\hline 7 & $\begin{array}{l}\text { Establecer cálculos para las huellas ambien- } \\
\text { tales }\end{array}$ & 3 \\
\hline 8 & Establecer el modelado de datos & 3 \\
\hline 9 & Establecer el diseño de base de datos & 5 \\
\hline 10 & Planificación y modelado de los procesos & 6 \\
\hline 11 & Gestionar encuestas & 6 \\
\hline 12 & Gestionar el catálogo de elementos & 6 \\
\hline 13 & $\begin{array}{l}\text { Gestionar el ingreso de variables para el } \\
\text { cálculo }\end{array}$ & 6 \\
\hline 14 & Planificación y modelado de los procesos & 3 \\
\hline 15 & Establecer usuarios & 3 \\
\hline 16 & Ingresos al sistema & 3 \\
\hline 17 & Permisos de usuario & 3 \\
\hline
\end{tabular}

Fuente: elaboración propia

- Administrador. Encargado de manejar la parte paramétrica de la aplicación; dado que la aplicación maneja su propia seguridad, es posible dividir estas opciones en varios roles de acuerdo con la especialidad que se requiera.

- Analista. Encargado de recolectar los requerimientos, las restricciones del usuario final y llevar esto a un lenguaje comprensible para el desarrollador y el administrador de bases de datos.

- Administrador de bases de datos. Diseñar, implementar y mantener el sistema de base de datos, así como establecer políticas y procedimientos relativos a la gestión, la seguridad, el mantenimiento y el uso del sistema de gestión de base de datos

- Desarrollador. Encargado de programar las funcionalidades críticas y no críticas de la aplicación.

- Tester de software. Encargado de diseñar, ejecutar e informar el resultado de las pruebas sobre la plataforma desarrollada. 


\subsubsection{Precondiciones}

- A fin de calcular el valor de huella, el usuario debe ingresar todos los datos de finca y de las labores propias del cultivo.

- La aplicación debe permitir visualizar el valor de huella de carbono e hídrica por labor agrícola.

\subsubsection{Catálogo de elementos}

- Departamento. Contiene la cantidad de regiones en la que está divido el país política y administrativamente.

- Municipio. División territorial administrativa en que se organiza un Estado; cada departamento tiene asociado un conjunto de municipios.

- Asociación. Es una organización que representa los intereses de un grupo de agricultores, de manera que un municipio puede tener varias asociaciones.

- Encuesta. Instrumento de caracterización que permite recolectar la información del agricultor, la finca y las labores agrícolas.

- Datos encuestados. Tabla que almacenará los datos generales del encuestado.

- Resultado proceso. Tabla que almacenará el valor de huella por cada práctica agrícola realizada en finca.

- Resultado total HC. Tabla que almacenará el valor de huella por cada lote de la finca y el total de la finca.

- Finca. Tabla que contiene las fincas de una asociación.

- Lote. Tabla que contiene los lotes de cada finca.

- Práctica agrícola. Son las diferentes actividades que se realizan en un lote al tomar como referencia el uso del software, y se clasifican así:

- Preparación del terreno: incluye destoconado, guadañada, subsolado, cincelado, arado y ahoyado.

- Establecimiento en campo: se refiere al proceso de siembra de las plántulas en el lote.

- Control de plagas o de enfermedades: es la aplicación de productos químicos o naturales para detener los daños causados en el cultivo.

- Control de herbáceas: se trata de impedir el desarrollo de cultivos diferentes a los sembrados por medios químicos, naturales o mecanizados.
- Aporcar: tierra que se acumula alrededor del tallo de la planta.

- Plateo: limpieza de herbáceas alrededor del tallo de la planta.

- Fertilización: suministro de nutrientes químicos o naturales al cultivo.

- Riego: suministro de una lámina de agua al cultivo en periodos de seguía prolongados.

- Podas: eliminación de material vegetativo sobrante en la planta para ayudar a formación de frutos.

- Cosecha: recolección de los granos maduros en el árbol.

- Poscosecha: labores hasta entregar el café al consumidor o la industria como materia prima.

- Transporte: para la movilización de los insumos que son suministrados al cultivo.

- Equipo: se utiliza para realizar las diferentes labores del cultivo puede ser manual o mecanizado. La pala, azadón, barra, machete, rastrillo y demás que tienen como energía al ser humano para realizar una labor son llamados manual. En el caso de requerir maquinaria como motores para fumigación, secadores, despulpadora, separador de café, lavadora de café, y pila pulidora.

- Variable: es el elemento que se mide al equipo cuando este es mecanizado.

- Tipo Labor: la labor se clasifica en manual o mecanizada.

- Usuario: persona que usará la plataforma.

- Rol: un rol es una colección de permisos definida para el sistema que se puede asignar a usuarios específicos.

La figura 1 describe las entidades necesarias para el modelado de datos que permita el cálculo de la huella de carbono e hídrica.

\subsection{Fase 2: desarrollo}

El software se trabaja utilizando la metodología ágil XP. Para la planificación y modelación de los procesos, se hará uso de Enterprise Architect, y como parte del software de implementación Framework JavaScript para interfaces gráficas de usuario, servidores de aplicaciones construido en Nodejs y motor de base de datos MySQL. 


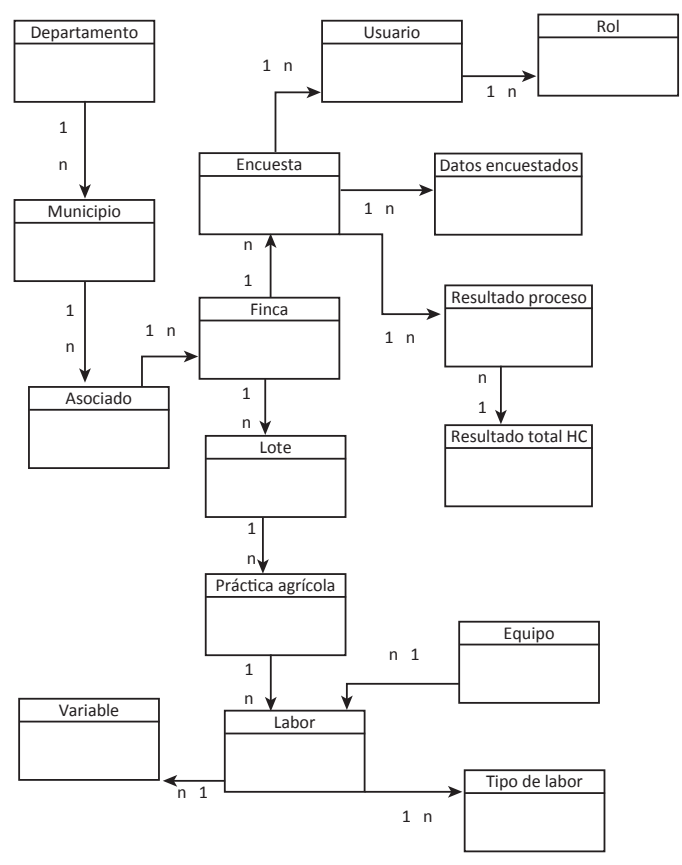

Figura 1. Modelado de datos

Fuente: elaboración propia

\section{Resultados}

La aplicación web para calcular huellas de carbono y huella hídrica durante el cultivo de café se desarrollará con la integración de tecnologías bajo licencia de software libre que permitan obtener eficiencia y eficacia en el desempeño. A fin de lograr este objetivo, se seleccionan cinco aspectos: escalabilidad, flexibilidad, adaptabilidad, interoperabilidad y modularidad. Las herramientas por utilizar se establecen de acuerdo con los siguientes ítems:

- Sistema de control de versiones. A fin de administrar el acceso a los ficheros y mantener un historial de cambios realizados, se utilizará GIT versión 2.10 [18]. Asimismo, se requiere hospedar el código fuente en un servidor de acceso remoto, para lo cual se cuenta con la plataforma de Github [19].

- Front-End. En la capa de presentación, se hace uso de HTML5 como lenguaje de estructura. Mediante el framework Bootstrap versión 3.3.7, se obtiene el diseño y la adaptabilidad de la aplicación [20]. Adicionalmente, se integra Angularjs versión 2 que es un framework orientado a crear SPA y tiene como objetivo lograr fluidez en Ux, así como Nodess [21]. Con modelo Single-Source-of-Truth, se logra que todo cambio visual se actualice en tiempo real en el modelo y viceversa. Por otra parte, con la ayuda de Json se logra el intercambio de datos.

- Back-End. A fin de programar la capa de negocio, se implementará Python versión 3.0 [22] que, junto con el framework Django versión 1.9, permite simplificar la programación mediante el principio DRY (dont repeat yourself) que consiste en mantener el código simple y no repetitivo. Django cuenta con un mapeo de objeto relacional ORM por defecto e integra la arquitectura MVT (model-template-view), la cual es una modificación del patrón Mvc con un sistema jerárquico de plantillas que favorece la reutilización de código y la extensibilidad de aplicaciones. De igual manera, ofrece la separación de responsabilidades entre las actividades realizadas por el programador y el diseñador sin colisionar.

- Gestor de base de datos. En la capa de datos, se plantea utilizar MySQL 5.7 [24], ya que su potencial en escalabilidad, eficiencia y rapidez en el procesamiento de datos - gracias al sistema multihilo y multiusuario- admite la sincronización de datos con gestores como SQLite [25], lo que permite integrar una aplicación móvil nativa para procesar los datos en fincas en las que no exista conexión a Internet.

\subsection{Arquitectura de la aplicación}

La arquitectura de la aplicación se refiere a la forma como el software seleccionado interactúa con los diferentes componentes para crear la calculadora de carbón e hídrica de forma estable, escalable y robusta. La figura 2 detalla la capa de presentación con HTML, Javascript, Bootstrap y Json para crear los marcos de trabajo, las conexiones a la base de datos y las validaciones, la capa de datos con ORM, Phythonmsqlconnector y MySQL Server para ejecutar lo relacionado con la base de datos y la capa de negocio Python, y deja con las vistas los modelos, el localizador de recursos uniforme URL, las formas y las plantillas a fin de crear los requisitos del cliente.

Con la integración de las herramientas mencionadas, se busca desarrollar una aplicación web que se adapte a dispositivos móviles y ofrezca eficiencia en la entrega de los datos. Asimismo, obtener una estructura organizada en el código fuente, lo que propicie un desarrollo ágil y flexibilidad a futuras modificaciones. 


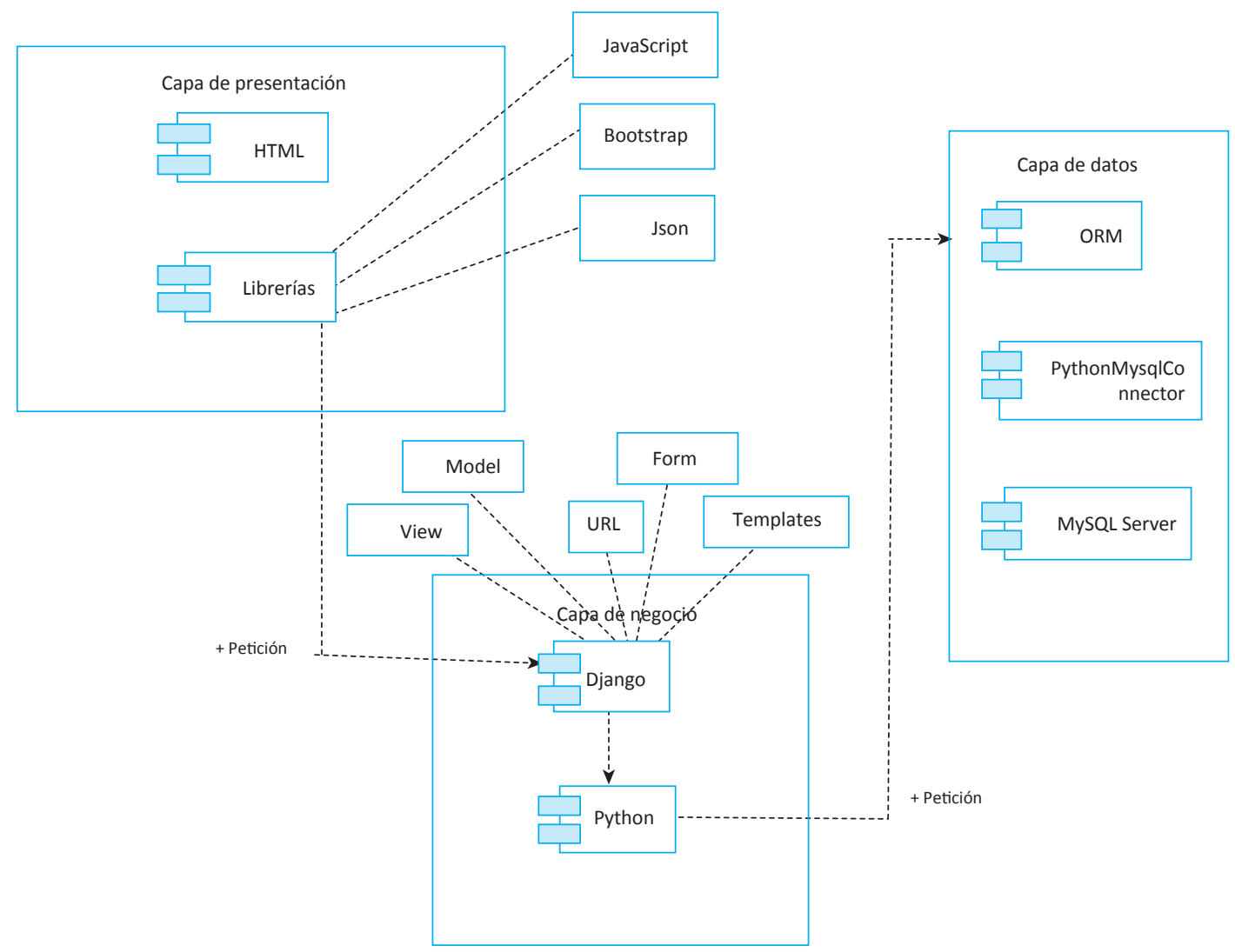

Figura 2. Arquitectura del software para el diseño de la aplicación de la calculadora de carbón e hídrica Fuente: elaboración propia

Para el logro del objetivo de "buscar una navegación fácil e interactiva de los usuarios con la plataforma web que contendrá las calculadoras, estableciendo una interface gráfica de buena calidad, teniendo presente factores como: usabilidad, accesibilidad, navegabilidad y portabilidad", se realizó un diseño web que contiene una página principal y las vistas de un CRUD ${ }^{1}$ en el que se encuentra "listar", "modificar", "adicionar", "consultar" y "encuestar", y permite su réplica en las demás tablas que se requieran en el proyecto (véase la figura 1). Está en proceso la adquisición del hosting para alojar el servicio web de la aplicación con las siguientes características: espacio en disco: 8GB; transferencia mensual: $80 \mathrm{~GB}$; correos electrónicos: ilimitados; cuentas FTP: ilimitadas; bases de datos: ilimitadas; y certificado de seguridad.

1 CRUD: create, read, update and delete ("crear, leer, actualizar y borrar").

\section{Conclusiones}

La aplicación de la metodología XP permite la descripción de las historias de los usuarios para el cálculo de la huella de carbón e hídrica de manera segura, rápida y dinámica. Los cambios se pueden ejecutar de forma inmediata sin necesidad de esperar a que terminen otras etapas del desarrollo del software, lo cual facilita la inclusión de nuevos estándares y requisitos que contribuyen a construir un software.

El proceso de investigación cualitativa y cuantitativa permite identificar variables involucradas en la producción del cultivo de café, finca, lote, control de herbáceas, plateo, fertilización y transporte, lo que contribuya al buen diseño para el desarrollo del software. Además de cumplir con estándares Greenhouse Gas Protocol Corporate Standard (GHg Protocol), UNE-ISO 14064-1, UNE-ISO 14065: 2012, IPCC 2006 GHG Workbook, Bilan Carbone 
(Francia), e indicadores GRI (Global Reporting Initiative e ISAE 3410).

La huella hídrica es un indicador de uso de agua directo, es decir, representa la cantidad de agua que hace falta para sostener la actividad de una población, con los componentes que se pueden especificar geográfica y temporalmente al tomar como referencia la huella hídrica de un proceso, la huella del agua verde, la huella del agua azul y la huella de agua gris.

El hecho de que los agricultores del sur del departamento del Huila cuenten con un software para el cálculo de la huella de carbón e hídrica les permite tomar decisiones con base en la manera como llevan a cabo las labores en el cultivo, con el fin de reducir las huellas y ofrecer al mercado internacional un café excelso y mejorar así los rendimientos económicos a los caficultores y reducir el impacto en el medio ambiente.

\section{Referencias}

[1] Café La Nacional, "Proceso de producción del café", Café la Nacional, dic. 5 2010. [En línea]. Disponible en: http://www.cafelanacional.com/nosotros/ proceso-de-produccion-del-cafe/

[2] Isagen, "Inventario de emisiones de gases de efecto invernadero", Isagen.com, 2 en. 2014. [En línea]. Disponible en: https://www.isagen.com.co/documentos/2015/inventario-de-emisiones-de-gases-de-efecto-invernadero.pdf

[3] Instituto Superior del Medio Ambiente, "Qué beneficios tiene el cálculo de la huella de carbono", 1 feb. 2012. [En línea]. Disponible en: http:// www. ismedioambiente.com/agenda/\%C2\%BFque-beneficios-tiene-el-calculo-de-la-huella-de-carbono

[4] Conservación \& Carbono, "Huella Hídrica", Conservacionycarbono.com, 1 mar. 2016. [En línea]. Disponible en: http://www.conservacionycarbono.com/ servicios/ huella-hidrica

[5] R. Martínez-Castillo, "Educación y huella ecológica", Revista Electrónica Actualidades Investigativas en Educación, vol. 8, no. 1, pp. 1-28, 2008. [En línea]. Disponible en: http://www.redalyc.org/articulo. oa? $\mathrm{id}=44780103$

[6] E. F. Viglizzo. Huella de carbono, ambiental y agricultura en el cono sur de Sudamérica. Argentina: INTA/ Conicet, 2010. [En línea]. Disponible en: https:// www.yumpu.com/es/document/view/14759104/ huella-de-carbon-ambiente-y-agricultura-en-el-cono-sur-procisur.
[7] Ministerio de Agricultura, Alimentación y Medio Ambiente. Guía para el cálculo de la huella de carbono y para la elaboración de una planta de mejora de una organización. Gobierno de España: 2016. [En línea]. Disponible en: http://www.mapama. gob.es/es/cambio-climatico/temas/mitigacion-politicas-y-medidas/guia_huella_carbono_tcm7379901.pdf

[8] Diario del Huila, "Por quinto año consecutivo, el Huila lidera producción de café en el país", Diariodelhuila.com, 18 nov. 2015. [En línea]. Disponible en: http://diariodelhuila.com/economia/por-quinto-ano-consecutivo-el-huila-lidera-produccion-decafe-en-el-pais-cdgint20151118190629194

[9] Diario del Huila, "En semestre A, producción de café en Huila creció el doble que en el país", Diariodelhuila.com, 7 jul. 2016. [En línea]. Disponible en: http:// www.diariodelhuila.com/economia/en-semestre-aproduccion-de-cafe-en-huila-crecio-el-doble-queen-el-pais-cdgint20160707123307177

[10] Asociación Española para la Calidad (AEC), "Huella hídrica”, AEC, 2 feb. 2017. [En línea]. Disponible en: https://www.aec.es/web/guest/centro-conocimiento/huella-hidrica

[11] I. Sánchez-Medina, F. Medina y J. M. Cabrera-Medina, "Software para el cálculo de la huella ambiental en la producción de cacao", Portal de Revistas UTP Memorias de Congresos, 2017, pp. 173-178. [En línea]. Disponible en: http://revistas.utp.ac.pa/index. $\mathrm{php} / \mathrm{memoutp} /$ article/view/1488/html.

[12] Water Footprint, "Manual para la evaluación de la huella hídrica", Waterfootprint.org, 2 feb. 2014. [En línea]. Disponible en: http://waterfootprint.org/media/downloads/ ManualEvaluacionHH.pdf

[13] Decreto 2667 [Ministerio del Ambiente y Desarrollo Sostenible]. Por el cual se reglamenta la tasa retributiva por la utilización directa e indirecta del agua como receptor de los vertimientos puntuales, y se toman otras determinaciones. 21 dic. de 1997. [En línea]. Disponible en: http://www.minambiente.gov.co/images/normativa/decretos/2012/ dec_2667_2012.pdf

[14] Minambiente, "Calculadora Huella Ecológica", Soyecolombiano.com, 2 en. 2015. [En línea]. Disponible en: http://www.soyecolombiano.com/huella-ecological

[15] Ecopetrol, "Calcule su huella de co2", Ecopetrol.com, 1 may. 2015. [En línea]. Disponible en: http://www. ecopetrol.com.co/especiales/calculadora Ambien$\mathrm{tal} / \mathrm{co} 2 . \mathrm{html}$

[16] Solidforest, "Descarga la versión de evaluación de Aire", Solidforest.com, 2 en. 2015. [En línea]. Disponible en: http://www.solidforest.com/software-airelca-demo.html 
[17] L. A. Rojas-Adames, F. Medina-Rojas, I. SánchezMedina y J. M. Cabrera-Medina, "Ingeniero de inclusión social: software administrativo para minimercados", Universidad Tecnológica de Panamá, 21 sep. 2016. [En línea]. Disponible en: http://revistas. utp.ac.pa/index.php/memoutp/article/view/ 1273/ html

[18] A. Freeman. Essential docker for ASP.NET Core MVC. Londres: Apress, 2017, pp. 150-200. [En línea]. Disponible en: https://www.apress.com/us/ book/9781484227770

[19] J. V. Talledo-San Miguel. Uf1467-Aplicaciones microinformáticas e Internet para consulta y generación de documentación. España: Paraninfo, 2016, pp. 30-82. [En línea]. Disponible en: http://www. paraninfo.es/catalogo/9788428396684/uf1467--aplicaciones-microinformaticas-e-internet-para-consulta-y-generacion-de-documentacion

[20] K. S. Prasad-Reddy. Beginning spring boot 2: applications and microservices with the spring. India: Apress, 2017, pp. 110-260. [En línea]. Disponible en: https://www.apress.com/la/book/9781484229309

[21] B. Dayley, J. S. Node, D. B. Mongo y J. S. Angular. Web development, usA: Addison-Wesley, 2014, pp.
39-75. [En línea]. Disponible en: https://www.amazon.es/Node-js-MongoDB-AngularJS-Development-Developers/dp/0321995783

[22] T. Hall y J.-P. Stacey. Python 3 for absolute begin ners. USA: Apress, 2010, pp. 130-146. [En línea]. Disponible en: https://www.apress.com/us/ book/ 9781430216322

[23] L. Debrauwer y Y. Evain. Patrones de diseño en PHP: los 23 modelos de diseño: descripciones y .... Barcelona: Eni Ediciones, 2015, pp. 220-280. [En línea]. Disponible en: https://www.casadellibro.com/libropatrones-de-diseno-en-php-los-23-modelos-de -diseno-descripciones-y-soluciones-ilustradas-enuml2-y-php/9782746098374/2672768

[24] P. DuBois, MysQL cookbook: solutions for database developers and administrators, Oreilly, UsA: 2014, pp. 335-401. [En línea]. Disponible en: https://www. amazon.es/MySQL-Cookbook-Solutions-Developers-Administrators/dp/1449374026

[25] S. Haldar. sQLite database system design and implementation. UsA: $2^{\text {a }}$ ed., Self-Publishing, 2015, pp. 83120. [En línea]. Disponible en: https://books.google. com.co/books?id=OEJ1CQAAQBAJ\&printsec=frontcover\&redir_esc $=\mathrm{y} \# \mathrm{v}=$ onepage $\& \mathrm{q} \& \mathrm{f}=$ false 